\title{
Longtime monitoring with smart sensor networks: the case of the Zwartewaterbrug
}

\author{
T. Siebel \& A. Friedmann \\ Fraunhofer LBF, Darmstadt, Germany \\ W. Strobel \& M. Guse \\ SWIFT GmbH, Reinheim, Germany
}

A. Hartmann

Uni Twente, Enschede, The Netherlands

\begin{abstract}
Experiences with a longtime monitoring system applied to the Zwartewaterbrug in the Netherlands are described in this paper. The investigated part is a steel tied-arch bridge of 104 meter in length. It was built in 1972 and is exemplary for the numerous aging bridges where real traffic loads exceed the design loads. The investigation focusses on testing the functionality of a prototype monitoring system. The proposed monitoring system is based on distributed vibration measurements and on the analysis of bridge modal parameters. The data acquisition unit consists of three sensor nodes and 12 measurement channels. Special attention is paid to the usability of low cost sensors for long term bridge monitoring. The monitoring system is simple to install, it stays permanently installed and acquires data during bridge operation. Measurement period was between October 2014 and June 2015. Results show that mode shapes and modal frequencies can automatically be identified and tracked while the bridge is in full operation.
\end{abstract}

\section{INTRODUCTION}

European road authorities are facing critical times. A huge number of bridges are now approaching their end of service life. Road authorities must cope with an increasing number of defects based e.g. on corrosion or fatigue obliging them to spend considerable resources for maintenance. In Germany for example the majority of road infrastructure was built in the years 1965 to 1985 . At the same time, the low design loads of the bridges during construction phase significantly deviate from today's high traffic loads (BMVI 2013).

Things get worse due to the small budgets road authorities dispose for maintaining their infrastructures.

Monitoring systems autonomously and periodically indicating the current health state of a bridge would be beneficial in two ways: the road authority would get a resilient basis for evaluating the real loads a bridge sustains. It further could include this quasi-real time information for optimizing its maintenance tasks.

Major challenges to monitoring systems are e.g. to deliver manageable amounts of data. Measurement data should thus be preprocessed in close to the data source, e.g. in the data logger. Furthermore, problems considering cabling and the reliable communicatoin for data transmission and control need to be fixed in order to use monitoring systems under real life conditions (Brownjohn 2007).

The majority of available bridge monitoring systems is based on local e.g. strain, vibration, or temperature measurements (Frost 2015). A drawback of these systems is that the damage location must be known a priori to installation. Slowly progressing damages affecting the global structure like change of foundation stiffness or boundary conditions can eventually not be detected.

In this paper a monitoring system for autonomous and periodical measurements is presented. It is characterized by a minimum of cabling and decentral data preprocessing. It analyzes the global vibration characteristics, i.e. the modal parameters, of a bridge. The monitoring system is permanently installed, works under traffic loads and yields periodic measurements of the bridges vibration characteristics.

\section{THE ZWARTEWATER BRUG}

The demonstration object is the Zwartewaterbrug which is located in the north-east of the Netherlands close to the town Hasselt, Figure 1. The bridge is owned and managed by the Province Overijssel. The bridge was built in 1972. It is composed of three sub-bridges: a steel arch bridge (104 m length), a bascule bridge (29 $\mathrm{m}$ length), and a pre-stressed concrete slab bridge (48 m length). Test object for 


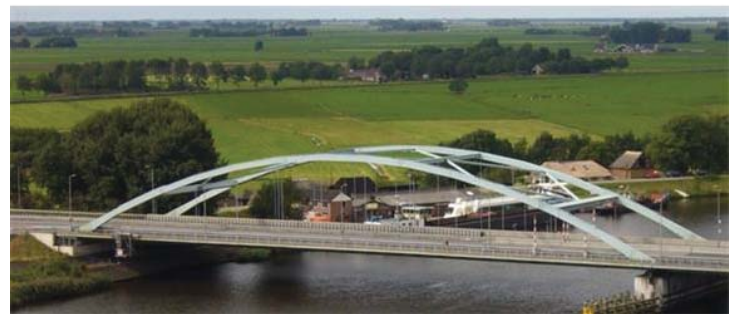

Figure 1: Zwartewaterbrug, Hasselt (NL),

(source: Z. Kasler, www.panoramico.com)

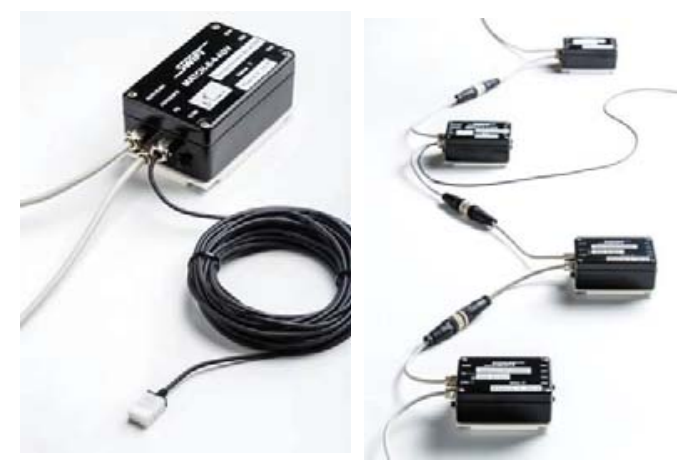

Figure 2: Smart sensor node and MEMS accelerometer (left), sensor nodes connected via CAN bus cable (right)

this investigation is the steel arch bridge. It is 25.4 meters in width and is crossed by two roads with two lanes each. The arch of the bridge is 13.3 meters at its highest position (wegenwiki 2016). Owner and operator of the bridge is Provincie Overijssel.

\section{SMART SENSOR NETWORK}

Measurements are conducted by the means of a network of smart sensor nodes and accelerometers, Figure 2, which can be flexibly arranged according to specific requirements of test structures and measurement task.

A sensor node is based on a $40 \mathrm{MHz}$ and 16 Bit microcontroller with internal flash for temporary data storage and CAN bus controller. It consumes less than $100 \mathrm{~mA}$ at a voltage of $5 \mathrm{~V}$. Different nodes are connected via one single cable for communication and power supply. A gateway node within the sensor network is connected to a central power supply and provides the power to all other network nodes. The gateway node contains a central data storage which is filled by data from the other nodes in defined intervals. Configuration of the sensor network is furthermore conducted at the gateway node. The smart sensor network is characterized by precise time synchronization with a maximum delay of approximately $1 \mu \mathrm{s}$. This is of particular importance for the time synchronous transfer and processing of trigger information between the individual nodes. The mission rate for a network extending to about 100 meter is $250 \mathrm{kBaud}$.

Vibration measurements are conducted by the means of MEMS accelerometers of the type LIS3DSH by ST Microelectronics. Scales between $\pm 2 \mathrm{~g}$ to $\pm 16 \mathrm{~g}$ can be measured with full 16 Bit resolution. The reason to use MEMS sensors is that they are significantly cheaper - however also less sensitive - than seismic sensors usually used in bridge monitoring.

In order to reduce the amount of data to be transferred in the network and to be stored on the central storage - and thus to allow for a minimum cabling effort - the measurement data is de-centrally preprocessed at each sensor node. The preprocessing is based on the Random Decrement (RD) technique.

\section{DATA PROCESSING}

The RD technique averages time data series $x\left(t_{n}\right)$ measured on a system subjected to random input loads. A measurement at all measurement channels is triggered when the amplitude at predefined trigger channel exceeds at a predefined trigger level $a$.

The result of the averaging process is the Random Decrement signature, which can be expressed by

$D_{x y}(\tau)=\frac{1}{N} \sum_{n=1}^{N} x\left(t_{n}+\tau\right) \mid y\left(t_{n}\right)=a$

with time series $x$ and $y$ and the length of the measured time interval $\tau$. Averaging a high number of time series the random part of the signal disappears and the result can be interpreted as the system response to the initial condition defined by the trigger. The RD signatures are related to the cross correlation functions $R_{x y}$ by the trigger level and the standard deviation $\sigma_{y}^{2}$ of the signal triggered (Brincker 1990):

$$
R_{x y}=D_{x y} \frac{\sigma_{y}^{2}}{a}
$$




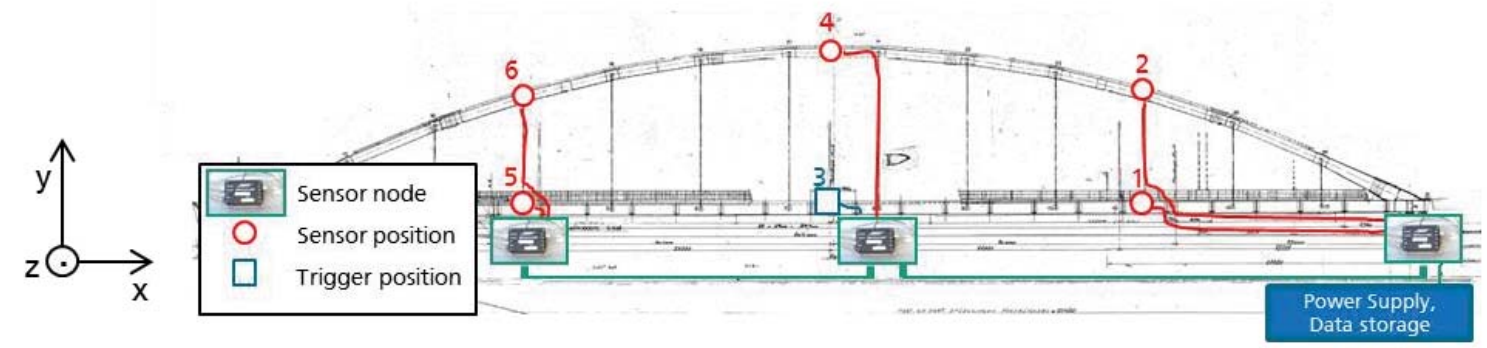

Figure 3: Sketch of the sensor network and according sensor position numbers. Sensor number 3 is the position of the trigger channels. Acceleration in $\mathrm{y}$ - and z-direction is measured at every sensor position

Auto correlation functions are estimated analogously. A set of all estimated auto and cross correlation functions is transferred to frequency domain by a Fast Fourier Transformation and is subsequently stored in a matrix of spectral densities $\boldsymbol{G}(f)$.

The spectral densities yield the basis for an operational modal analysis (OMA). An OMA is an output-only structure identification technique. This means that only measured output signals of structural vibrations are used for analysis while the input signals, e.g. excitation force, is unknown.

In this investigation the Frequency Domain Decomposition technique is applied as OMA method. Here, the spectral density matrix $\boldsymbol{G}(f)$ resulting from the correlation matrix is subjected to a Singular Value Decomposition

$\boldsymbol{G}(f)=\boldsymbol{U}(f) \boldsymbol{S}(f) \boldsymbol{U}^{H}(f)$

which leads to fully populated matrices $\boldsymbol{U}(f)$ and a diagonal matrix $\boldsymbol{S}(f)$ holding the spectra of the singular values (SV). The peak values of the first singular values are interpreted as indicators for the system's modal frequencies. The estimated mode shapes are found as the first column of $\boldsymbol{U}(f)$ according the modal frequencies. A detailed description of the procedure can e.g. be found in (Friedmann 2010).

An important role in automating the analysis process is automated selection of mode shapes of current data set cur that correspond to mode shapes in a baseline data set base. The selection is based on Modal Assurance Criterion (MAC) analysis. A MAC value between two sets of modal vectors $\left\{U_{\text {cur }, m}\right\}$ and $\left\{U_{\text {base }, m}\right\}$ is calculated by

$M A C_{c u r, d, m}=\frac{\left|\sum_{d=1}^{N}\left\{U_{\text {cur }, d, m}\right\}^{H}\left\{U_{\text {base }, d, m}\right\}\right|^{2}}{\left\{U_{\text {cur }, d, m}\right\}^{H}\left\{U_{\text {cur }, d, m}\right\}\left\{U_{\text {base }, d, m}\right\}^{H}\left\{U_{\text {base }, d, m}\right\}}$

with of mode number $m$. The MAC is a scalar related to the consistency - or orthogonality - between one modal and another modal vector (Allemang
2003). The MAC takes values between zero and one, while zero means no consistency between the modal vectors and one means full constancy.

The MAC analysis plays a vital role in the automated analysis of the measurement data. First a baseline measurement is defined and the respective mode shapes are calculated. All calculated mode shapes of subsequent measurements are then compared to the baseline mode shapes by the means of a MAC analysis. Only if baseline and currently measured mode shapes reveal a MAC value close to one they are considered similar and are used for further analysis.

The MAC value is furthermore used for monitoring the structure. A damage to load carrying parts of the structure resulting in significant changes in stiffness or mass distribution would lead to a decrease in the MAC values.

\section{INSTALLATION AND TEST SETUP}

In order to create a long-term monitoring system the data acquisition system has been permanently installed to the bridge. Two workers have applied the system within approximately half a day while one lane of the bridge was closed.

Within this investigation one side of the steel arch bridge should be subjected to a monitoring system. The sensor network for this is arranged according to Figure 3. Three sensor nodes are distributed over the length of the bridge deck. The gateway node is located close to a bridge pile. The bridge pile, and thus the central data storage, is easily accessible and provides the power supply for the sensor network. Acceleration is measured by the means of six biaxial accelerometers, each measuring vibration in vertical $\mathrm{y}$-, and the horizontal z-direction (orthogonal to the road direction).

The two channels of the centrally located sensor at position 3 are defined as trigger channels. This means that every time acceleration in the respective direction exceeds a defined trigger level all sensors of the network are triggered to record measurements for the time of 20.48 seconds. Single measurements 
within one averaging interval may overlap. The trigger levels are configured such that trucks passing over the bridge trigger a measurement. These and other configuration parameters can be taken from Table 1.

Table 1: Configuration of the data acquisition

\begin{tabular}{ll}
\hline Parameters & Values \\
\hline Number of measurement channels & 12 \\
Number of sensor nodes & 3 \\
Measurement range & $\pm 1.5 \mathrm{~m} / \mathrm{s}^{2}$ \\
Sampling rate & $100 \mathrm{~Hz}$ \\
Sample number & 2048 \\
Trigger level (y) & $0.2 \mathrm{~m} / \mathrm{s}^{2}$ \\
Trigger level (z) & $0.3 \mathrm{~m} / \mathrm{s}^{2}$ \\
Averaging time & $60 \mathrm{minutes}$
\end{tabular}

It must be stated that time for configuration of the sensor network was limited and during the evening after rush hour. Parameters could not be adjusted during the monitoring period. The sensor nodes average the measurements during a time span of 60 minutes, i.e. every 60 minutes a $\mathrm{RD}$ signature at each sensor channel is completed. Every 60 minutes the sensor nodes transfer the resulting RD signatures to the gateway node where they are stored. Hence, in a later analysis the vibration characteristic of the bridge arch can be evaluated once per hour. Approximately once every two months the stored data is manually transferred to a central computer for further analyzes.

\section{RESULTS}

Measurements have been conducted between 2 October 2014 and 16 June 2015. Figure 4 shows the number of trigger events per hour for the two trigger channels for the week between Monday, 6 October and Sunday, 13 October. Each time stamp in the diagram indicates a new day. The diagram is exemplary for the number and distribution of trigger events during a typical work week. It can be observed that the number of triggers in z-direction is lower than in y-direction. For this investigation this is not regarded as critical, however would be a point to approve in a subsequent study. The distribution of trigger events further indicates day / night and week day /week end changes. Since the trigger levels are adjusted to vibration levels typically induced by heavy weight traffic, the number of trigger events is highest between Monday and Friday and between 8:00 am and 6:00 pm.

As baseline measurement is selected the measurement conducted on Monday, October $6^{\text {th }} 2014$ between 9:00 and 10:00 am. This measurement is close to the beginning of the data acquisition period and

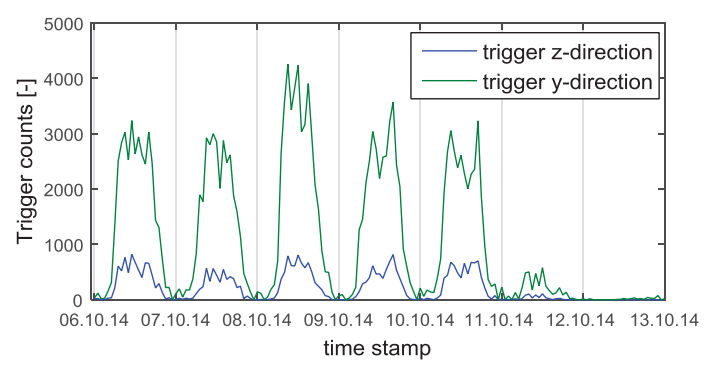

Figure 4: Trigger events at two trigger channels from Monday $6^{\text {th }}$ to Sunday $13^{\text {th }}$ October

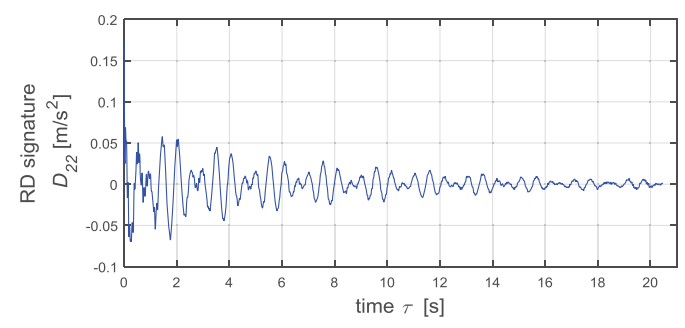

Figure 5: RD signature of trigger channel,

vertical direction

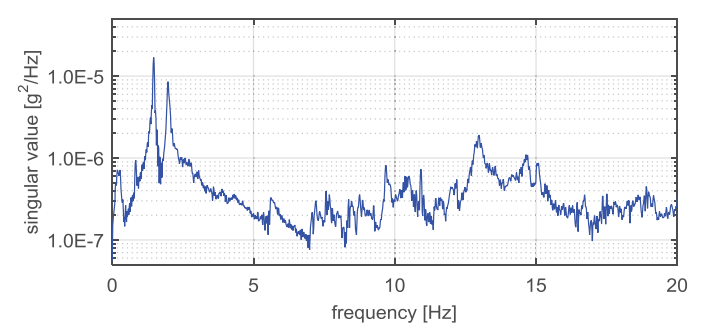

Figure 6: Spectrum of first singular value for baseline measurement

traffic loads at this time are representative for most of the weekdays between Monday and Friday.

An example for a RD signature is shown in Figure 5. It is measured at the trigger node in vertical (y) direction during the baseline measurement. Within the 60 minutes of the baseline measurement, this trigger experienced 2842 trigger events. This means that 2842 times measurements of 20.48 seconds have been averaged to generate this RD signature.

Twelve RD signatures in total are generated during one measurement period - one at each of the twelve sensor channels. The set of RD signatures is subsequently converted to auto and cross correlation functions and next transferred to frequency domain by a Fast Fourier transformation. 

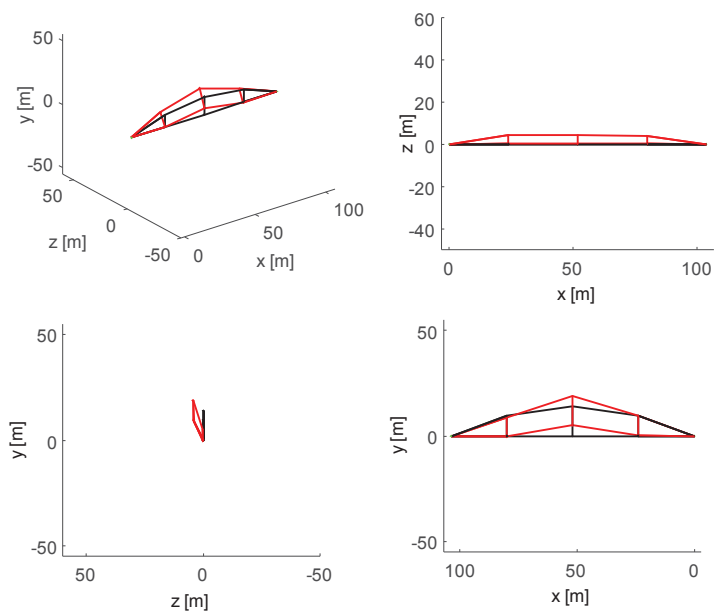

Figure 7: $1^{\text {st }}$ selected baseline mode shape at $1.46 \mathrm{~Hz}$ (deformed and undeformed structure )
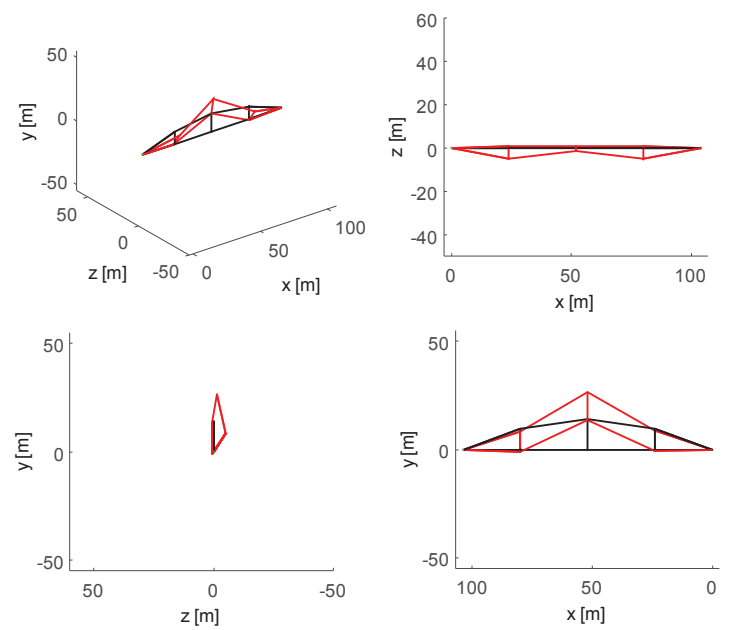

Figure 8: $2^{\text {nd }}$ selected baseline mode shape at $1.96 \mathrm{~Hz}$ (deformed and undeformed structure )
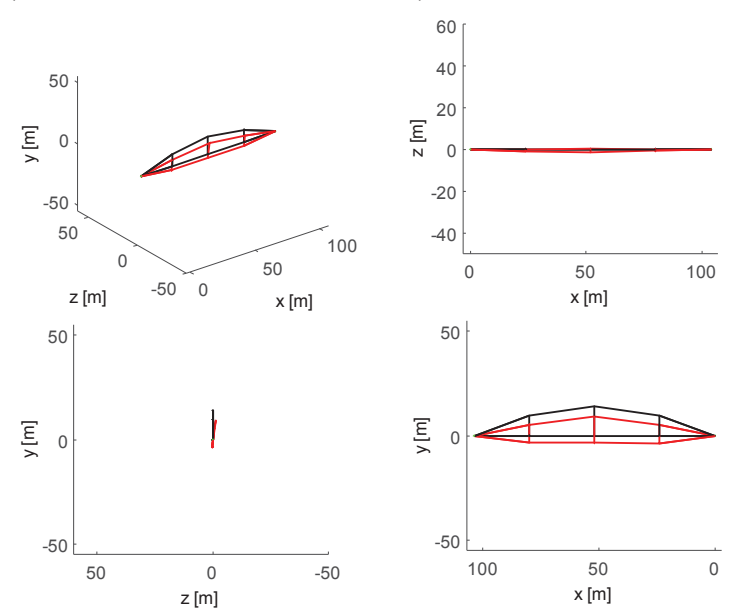

Figure 9: $3^{\text {rd }}$ selected baseline mode shape at $12.97 \mathrm{~Hz}$ (deformed and undeformed structure )
During the setup phase for the monitoring system a number of baseline mode shapes are selected to be tracked during the monitoring phase. Figure 6 shows the spectrum of the first singular value of the baseline measurement. The local maxima indicate the positions of modal frequencies. Peaks at $1.46 \mathrm{~Hz}$, $1.96 \mathrm{~Hz}$ and $12.97 \mathrm{~Hz}$ and their corresponding modal frequencies and mode shapes are selected as baseline data. An analysis of subsequent measurement intervals justifies this selection, because these three modes are found in the most of the data sets, they are reproducible under similar excitation conditions and thus reflect characteristic vibration of the bridge.

Figure 7 to Figure 9 illustrate the selected baseline mode shapes. The first baseline mode shapes at $1.46 \mathrm{~Hz}$ is a sideways tilt of the arch. At $1.96 \mathrm{~Hz}$ the arch center shifts vertically while the eccentrical parts of the arch tilt synchronously in horizontal direction. Furthermore a bending mode shape in vertical direction at $12.97 \mathrm{~Hz}$ is selected. This $3^{\text {rd }}$ selected mode shape resembles a $1^{\text {st }}$ bending mode of a beam type structure, however, in this case this appearance is due to the small number of available sensors.

Data processing in the monitoring phase is automated including the process of picking peaks in the SV spectrum, conducting operational modal analysis, and identifying mode shapes in a current data set that correspond to the baseline mode shapes. The algorithm to automatically select local maxima of the SV spectrum typically generates a number of mode candidates that exceeds the true number of modes within the frequency range. By the means of a MAC analysis all mode candidates are analyzed for consistency to the baseline modes. Only the modes with the highest consistency, i.e. MAC value closest to one, are taken into account for the further monitoring process.

Figure 10 shows the MAC values for the complete monitoring phase between October 2014 and June 2015. Every point in the diagram corresponds to the maximum MAC value within one measurement for a specific mode shape. The plot shows that the selected baseline mode shapes can be tracked over a period of several months with the proposed monitoring system. Although there is scatter within MAC values most of the automatically identified mode shapes correspond to the baseline mode shapes with a MAC value of significantly higher than 0.95.

The diagram however also reveals some exceptions. Around the $25^{\text {th }}$ of October the MAC values of all three mode shapes decrease for a period of about four days. The same can be observed for the period between $5^{\text {th }}$ January and $19^{\text {th }}$ January. Reasons to explain this behavior might be changing traffic conditions or climate induced changes to the structure. However, within this investigation no traffic or weather data has been acquired, so that these effects have not been analyzed. 


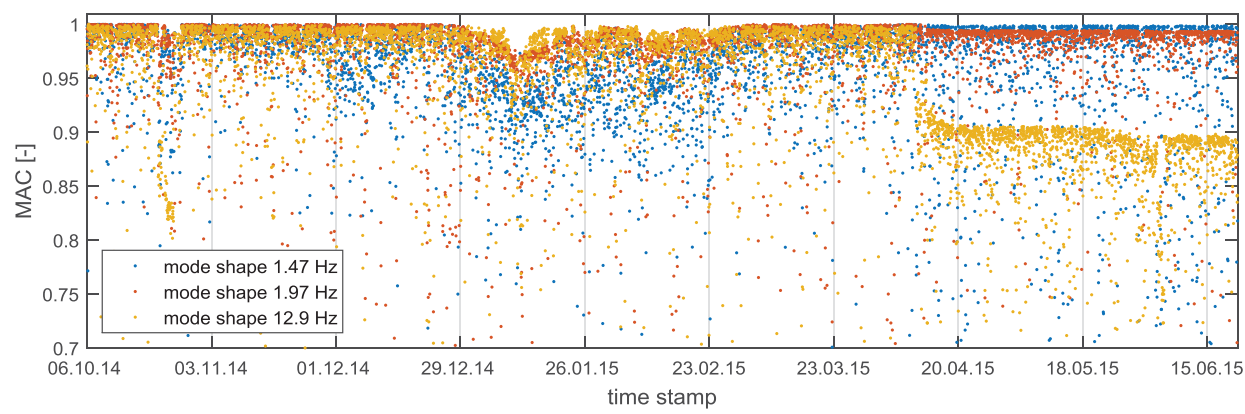

Figure 10: MAC vs. time plot

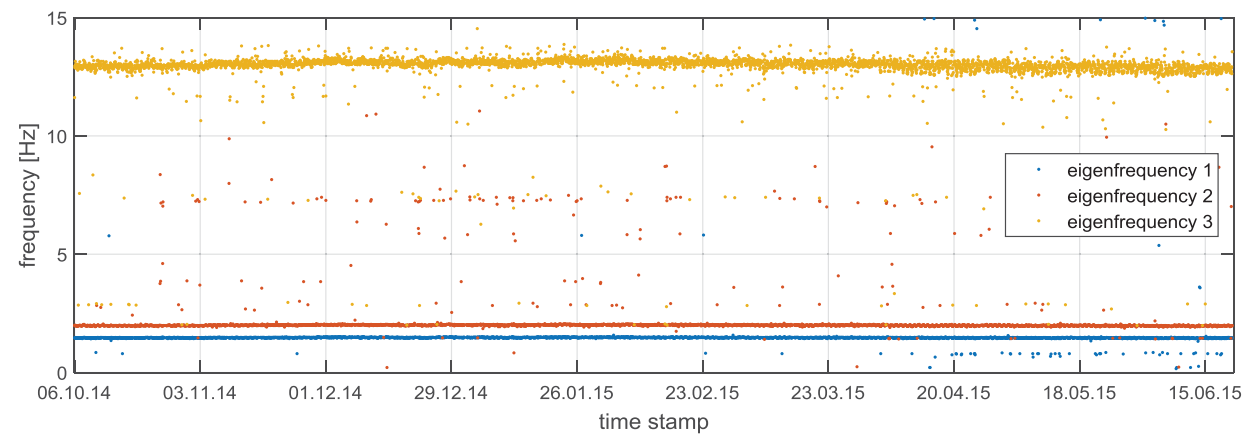

Figure 11: Modal frequency vs. time plot

Beginning from $11^{\text {th }}$ April the MAC value of the $12.9 \mathrm{~Hz}$ mode shape significantly drops. As a reason for that a defect sensor at position 1 in vertical $y$ direction has been identified. The MAC value of the selected mode shape at $12.97 \mathrm{~Hz}$ is particularly impacted by the defect, since this mode shape describes a pure vertical deflection. The vertical deflection of position 1 only little contributes to the mode shapes at $1.46 \mathrm{~Hz}$ and $1.96 \mathrm{~Hz}$ and thus the corresponding MAC values are hardly impacted by the sensor defect.

Figure 11 shows the modal frequencies corresponding to the identified mode shapes for the complete monitoring period. The representation of the same data in Figure 12 shows illustrates the seasonal variations of the frequencies: The frequencies increase during the winter months.

\section{CONCLUSION AND OUTLOOK}

A monitoring system consisting of smart sensor nodes, low cost accelerometers and a data processing routine based on automated operational analysis and tracking of modal parameters has been proposed in this paper. The monitoring system has been installed and run on the Zwartewaterbrug for more than seven months. The major intention of this investigation was to proof if this configuration of a monitoring system is capable to do structure identification and to track the identified parameters for a period of several months under real world conditions. As shown in the results this target was reached within the investigation.

However, the investigation also revealed a number of issues to be addressed in future work with the monitoring system. Future investigations will take into account the different operational and environmental conditions the bridge is subjected to. I.e. it is necessary to define a broader set of baseline parameters covering the whole spectrum of external conditions.

Due to lower sensitivity of the used MEMS accelerometers compared to seismic sensors - particularly in the frequency range up to $1 \mathrm{~Hz}$ - relevant mode shapes of the bridge structure are not identified. It has to be evaluated in which cases the monitoring of a few identifiable mode shapes with low cost sensors is beneficial over an expensive but more precise monitoring with seismic sensors. In all other cases the sensor network can also be used with high precision seismic sensors.

Another issue is the small number of sensors used for this investigation. Especially for the mode shape at $12.97 \mathrm{~Hz}$ it is obvious that a denser distribution of sensors is required to fully identify the real deflection of the mode shape. 

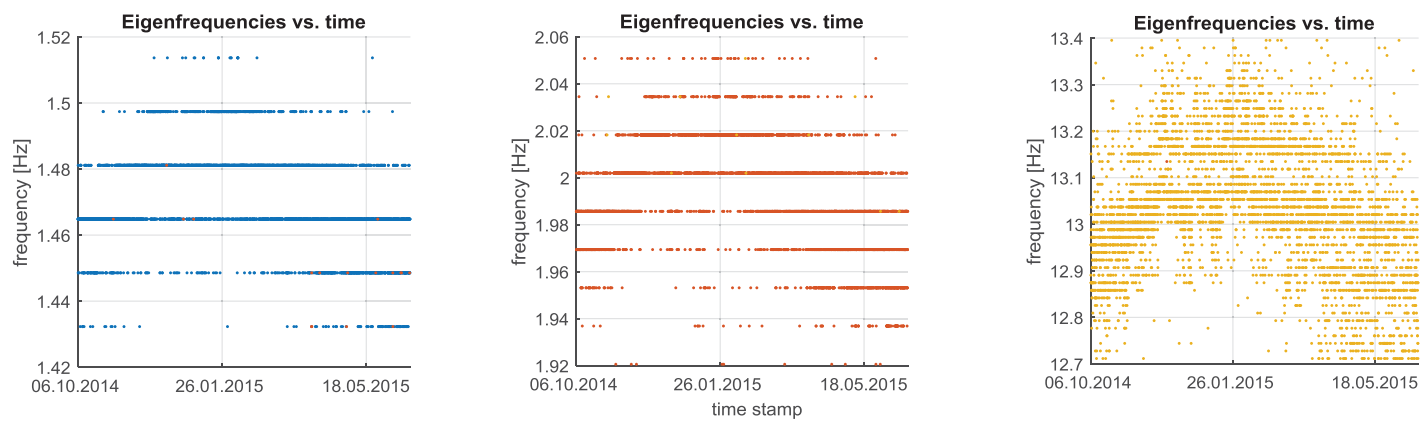

Figure 12: Modal frequencies and seasonal variations

As seen in Figure 5 the number of trigger events per trigger channel and per measurement deviates significantly. Due to limited project budget it was not possible to reconfigure the monitoring system. This will be considered in future projects.

A major issue for the further development of the monitoring system is the consistency of the automated data processing from sensing level to data interpretation. In this investigation data was stored centrally on a gateway node located at the bridge and had to be manually transferred every few weeks. A future version of the monitoring system will mesh the data acquisition and analysis.

Future investigations will furthermore address the dependence between vibration characteristics and effects of climate and traffic conditions.

The proposed monitoring system is based on the identification of global vibration characteristics. It is thus appropriate for monitoring of parts within the major load paths or the boundary conditions of a bridge structure. A benefit is that potential damage locations must not be known a priori to the planning of sensor locations. A further field of application for the proposed monitoring system is to deliver data for adjusting or updating numeric bridge models. This is simplified by the fact that the data acquisition hardware is scalable to specific requirements of a bridge and easy to install.

\section{ACKNOWLEDGEMENTS}

The results presented in this contribution were partly funded by the LOEWE-Zentrum AdRIA (Adaptronic - Research, Innovation, Application) coordinated by Fraunhofer LBF and funded by the government of the German federal state Hesse. The support is greatly acknowledged.

\section{REFERENCES}

Allemang, R. J. "The modal assurance criterion-twenty years of use and abuse." Sound and vibration 37.8 (2003): 14-23.

Brincker, R., Krenk, S., Jensen, J. L., Estimation of Correlation Functions by the Random DEC Technique. Dept. of Building Technology and Structural Engineering, Aalborg University, 1990

Brownjohn, J. M. W., "Structural health monitoring of civil infrastructure." Philosophical Transactions of the Royal Society of London A: Mathematical, Physical and Engineering Sciences 365.1851 (2007): 589-622.

Bundesministerium für Verkehr, Bau und Stadtentwicklung (BMVI), Bauwerksprüfung nach DIN 1076 Bedeutung, Organisation, Kosten, Dokumentation 2013

Friedmann, A., Koch, M., Mayer, D., Using the Random Decrement Method for the Decentralized Acquisition of Modal Data, Proceedings of ISMA2010, September 2010

Frost \& Sullivan, Analysis of the Global Sensors in Infrastructure Monitoring and Smart Buildings Market, Connectivity Empowers Sensors to Monitor Infrastructure and Buildings, NF88-32, September 2015

https://www.wegenwiki.nl/Zwartewaterbrug, status: 14.04.2016 